\title{
Um Estudo sobre a Influência das Dimensões do Modelo Felder-Silverman na Recomendação de Recursos Educacionais baseada nos Estilos de Aprendizagem dos Alunos
}

\author{
Janderson Jason Barbosa Aguiar ${ }^{1}$, Anderson Felinto Barbosa ${ }^{2}$, \\ Joseana Macêdo Fechine Régis de Araújo ${ }^{1}$, Evandro de Barros Costa ${ }^{3}$ \\ ${ }^{1}$ Universidade Federal de Campina Grande (UFCG), Campina Grande - PB - Brasil \\ ${ }^{2}$ Instituto Federal da Paraíba (IFPB), Campus Monteiro - PB - Brasil \\ ${ }^{3}$ Universidade Federal de Alagoas (UFAL), Maceió - AL - Brasil \\ janderson@copin.ufcg.edu.br, anderson.barbosalifpb.edu.br, \\ joseana@dsc.ufcg.edu.br, evandro@ic.ufal.br
}

\begin{abstract}
It is possible to use the theory of Learning Styles to build the user profiles in Recommender Systems for Learning (RSL). This paper presents a study about the influence of the four dimensions of the Felder-Silverman Learning Styles Model (FSLSM) in the construction of user profiles in RSL. For this, we analyzed the possibility of using only some dimensions to simplify this construction, while maintaining accurate personalized recommendations. The results showed that the dimensions of the FSLSM do not have the same influence on the recommendation and that - although it has not been possible to highlight precisely those of the greatest influence - there are indications that the use of half of the dimensions is sufficient.
\end{abstract}

Resumo. A teoria dos Estilos de Aprendizagem (EA) pode ser utilizada para construir o perfil dos usuários em Sistemas de Recomendação Educacionais (SRE). Neste artigo é apresentado um estudo sobre a influência das quatro dimensões do modelo de EA de Felder-Silverman (FSLSM) na construção de perfis de usuários de SRE. Para isso, analisou-se a possibilidade de utilizar apenas alguma(s) dimensão(ões) para simplificar esta construção, mantendo, ainda, as recomendações personalizadas acuradas. Os resultados apontaram que as dimensões do FSLSM não têm a mesma influência na recomendação e que, embora não tenha sido possível destacar precisamente aquelas de maior influência, há indícios de que o uso de metade das dimensões seja suficiente.

\section{Introdução}

A busca por melhorias na recomendação personalizada de conteúdo no contexto educacional é pesquisa corrente. Os alunos têm necessidades individuais, sendo importante reconhecer, portanto, que cada um tem sua própria maneira de receber e processar informações, resolver problemas e expor ideias.

Identificar os Estilos de Aprendizagem (EA) dos alunos é uma das formas para a obtenção de suas preferências, propiciando materiais de aprendizagem mais efetivos no processo de ensino-aprendizagem [Aguiar, Fechine e Costa 2014] [Valaski, Malucelli e Reinehr 2011]. Os EA se referem aos modos a partir dos quais os indivíduos preferem receber e processar informações. Dentre os modelos existentes, destaca-se o modelo 
VI Congresso Brasileiro de Informática na Educação (CBIE 2017)

Anais do XXVIII Simpósio Brasileiro de Informática na Educação (SBIE 2017)

Felder-Silverman [Felder e Silverman 1988], amplamente utilizado em pesquisas relativas à adaptação e personalização de materiais de aprendizagem.

Nesse contexto, julgou-se relevante realizar este estudo, por empregar EA para buscar melhorias na construção do perfil dos discentes (visando a uma acurácia maior na seleção personalizada de itens) em Sistemas de Recomendação Educacionais (SRE).

O objetivo do estudo realizado concentra-se em investigar o impacto de utilizar apenas alguma(s) dimensão(ões) do modelo Felder-Silverman para a construção de um perfil simplificado dos usuários (discentes) de SRE. Nesse sentido, foram consideradas as seguintes questões de pesquisa: As dimensões de EA do modelo Felder-Silverman têm a mesma influência na recomendação personalizada de recursos educacionais para discentes? É possível destacar a(s) dimensão(ões) com maior influência nesse processo de recomendação?

O restante do artigo está estruturado da seguinte forma: na Seção 2, são comentados conceitos e estudos relacionados; na Seção 3, é descrito o método empregado no estudo; na Seção 4, são mostrados e discutidos os resultados; e, na Seção 5, são apresentadas as considerações finais.

\section{Referencial Teórico}

Sistemas de Recomendação (SR) são úteis para retornar conteúdos mais específicos aos usuários, permitindo, assim, encontrar algo que realmente lhes interessa, mais apropriado ao seu perfil [Aggarwal 2016]. Dentre as técnicas utilizadas em SR, uma das mais conhecidas é a Filtragem Colaborativa (FC), sendo também uma das mais empregadas em SRE. Na FC, a partir de avaliações realizadas pelos usuários do sistema, é possível recomendar os itens melhor avaliados por usuários com preferências similares, não sendo preciso conhecer as características dos itens [Costa, Aguiar e Magalhães 2013].

Uma vez que os SRE diferem de outros SR devido a considerar características educacionais, muitas vezes são estudados aspectos pedagógicos e psicológicos visando serem incorporados no sistema para prover melhores recomendações.

Em Psicologia e em Educação, as denominadas Teorias da Aprendizagem (TA) propõem-se a explanar o processo de aprendizagem pelos indivíduos. A literatura apresenta diversas TA que dão suporte a diferentes perspectivas educacionais [Schunk 2012] [Siemens 2005]. Segundo Bativa e Stiubiener (2011), as definições dos EA foram influenciadas pelas TA, uma vez que é possível reconhecer algumas características dos alunos esperadas pelos EA nas TA. A TA Behaviorista, por exemplo, defende o aluno como receptor das informações entregues pelo professor, por outro lado, a TA Construtivista mostra o aluno participando da construção do conhecimento, relacionamento este com experiências anteriores e, além disso, podendo compartilhar o que foi construído para outros alunos, colaborando, assim, para um aprendizado de forma colaborativa [Schunk 2012]. Contudo, ressalta-se que não há um mapeamento um-para-um entre EA e TA, pois diferentes características que são esperadas dos alunos nas TA podem ser encontradas em diferentes EA e vice-versa.

O modelo Felder-Silverman, citado na Seção 1, contempla quatro dimensões de EA: (1) Processamento - estilos Ativo e Reflexivo; (2) Percepção - estilos Sensorial 
VI Congresso Brasileiro de Informática na Educação (CBIE 2017)

Anais do XXVIII Simpósio Brasileiro de Informática na Educação (SBIE 2017)

e Intuitivo; (3) Entrada - estilos Visual e Verbal; e (4) Compreensão — estilos Sequencial e Global. Detalhes podem ser obtidos em [Felder e Silverman 1988].

Segundo Klašnja-Milićević et al. (2017), é óbvio que diferentes alunos têm diferentes EA, sendo importante considerá-los nos ambientes de aprendizagem que tais alunos estão inseridos. Com o conhecimento dos diferentes EA, os sistemas adaptativos de e-learning podem oferecer valiosos conselhos e instruções a alunos e professores, para otimizar o processo de aprendizagem dos alunos [Truong 2016].

$\mathrm{Na}$ literatura, há diversos estudos relativos à recomendação de recursos educacionais baseada em EA, a exemplo das pesquisas de Zaina et al. (2012), Resende e Dorça (2015) e Aguiar, Fechine e Costa (2015). Nessas pesquisas, em geral, são consideradas no processo de recomendação todas as quatro dimensões do modelo Felder-Silverman, com exceção da pesquisa de Zaina et al. (2012), que não considerou a dimensão Compreensão (o motivo foi que, segundo os autores, o conteúdo usado nos experimentos não abarcaria a complexidade de tal dimensão; ou seja, não foi devido a algum estudo sobre o pouco impacto desta dimensão no processo de recomendações).

Ressalta-se que, no contexto nacional, foi realizada uma busca - utilizando "Felder" como string, por ser um termo abrangente - nos engenhos de busca de veículos importantes de divulgação de produção científica brasileira em Informática na Educação: SBIE (Simpósio Brasileiro de Informática na Educação), WIE (Workshop de Informática na Escola), RBIE (Revista Brasileira de Informática na Educação) e IETP (Informática na Educação: Teoria \& Prática). Além dessa busca, foram analisados os artigos resultantes do mapeamento sistemático realizado por Aguiar, Fechine e Costa (2014), sobre iniciativas brasileiras relativas ao uso de EA em Informática na Educação. Entretanto, não foram encontradas pesquisas publicadas com objetivo similar ao deste artigo (citado na Seção 1). Isso reforça a escassez de estudos relativos às questões de pesquisa levantadas.

Uma vez que os EA podem ser considerados um componente da personalidade de um indivíduo [Klašnja-Milićević et al. 2017], é conveniente citar que há estudos com resultados indicando maior eficácia e lealdade dos usuários em sistemas baseados em personalidade do que em sistemas não baseados em personalidade [Hu e $\mathrm{Pu} 2011$ apud Roshchina 2012] [Elahi et al. 2013] [Xia et al. 2014].

No contexto de personalidade, é interessante destacar a pesquisa de Roshchina (2012), que se assemelha a este por também estudar o impacto de desconsiderar algumas dimensões/partes de um modelo de personalidade (Big Five) na construção do perfil de usuários em SR usando FC (experimentos com dados no domínio de turismo).

Considerando os conceitos e estudos citados nesta seção, julgou-se relevante realizar o estudo descrito nas seções seguintes.

\section{Método}

Para obter respostas às questões de pesquisa, foi planejado um experimento com um conjunto de dados referentes a 55 participantes (alunos de graduação e pós-graduação em Ciência da Computação), contemplando seus EA (obtidos a partir do ILS - Index of Learning Styles [Felder e Soloman 1999], baseado no modelo Felder-Silverman) e suas notas (avaliações em uma escala de 1 a 5) para 25 itens (variações de características de Objetos de Aprendizagem, segundo o padrão LOM [IEEE 2002]). 
Para considerar o impacto das dimensões de EA nas recomendações, foi empregada a abordagem de FC, utilizando-se da Distância Euclidiana para calcular a similaridade entre os perfis dos usuários (cada perfil de usuário foi construído com base em vetores numéricos de tamanho até 4, com os valores percentuais para cada dimensão de EA). Foram variados os tamanhos dos vetores representativos dos usuários, retirando valores para analisar se alguma dimensão (ou conjunto de dimensões) se destacava em relação a considerar todas as dimensões do modelo Felder-Silverman.

A partir disso, foram comparadas as 15 variações do algoritmo de recomendação baseada em EA, nomeados como: LSBR_FC-d1, LSBR_FC-d12, LSBR_FC-d123, LSBR_FCd1234, LSBR_FC-d124, LSBR_FC-d13, LSBR_FC-d134, LSBR_FC-d14, LSBR_FC-d2, LSBR_FCd23, LSBR_FC-d234, LSBR_FC-d24, LSBR_FC-d3, LSBR_FC-d34, LSBR_FC-d4. O termo LSBR foi usado como sigla para Learning Style Based Recommendation. Já os números após a letra d (1, 2, 3 e/ou 4) representam as dimensões contempladas em cada variação, sendo a correspondência entre esses números e as dimensões apresentada na Seção 2.

Adicionalmente, foi comparado o desempenho dessas variações com o desempenho de uma abordagem tradicional em SR, o UserKNN (User based K-Nearest Neighbors). Uma vez que foi utilizada a estratégia de FC com base nos vizinhos mais próximos, além de variar as dimensões de EA, decidiu-se, no experimento, variar o valor de $\mathrm{K}$, pois se percebeu que a quantidade de vizinhos, para a base de dados adotada, influenciava o resultado da recomendação. Foram feitas análises baseando-se nos seguintes valores de $\mathrm{K}: 5,10,15,20$ e 25 .

Para a obtenção dos resultados, empregou-se o método Random Subsampling variação do método Holdout [Han e Kamber 2006] —, sendo separados aleatoriamente, por 100 vezes, os dados em dois conjuntos: 2/3 dos dados para o conjunto de treinamento dos algoritmos de recomendação, e 1/3 dos dados para o conjunto de testes. As variáveis dependentes deste experimento consistiram em métricas relacionadas à acurácia da recomendação: AUC (Area Under ROC Curve), MAP (Mean Average Precision), NDCG (Normalized Discounted Cumulative Gain), P@5 (Precision at 5) e R@5 (Recall at 5) [Gantner et al. 2011] [Aggarwal 2016]. Todos os intervalos de confiança gerados e os testes estatísticos realizados consideraram $\alpha=0,05$ (ou seja, 95\% de confiança).

\section{Resultados e Discussões}

Com base no método descrito na seção anterior, foi elaborada a Figura 1, para uma análise inicial, na qual se apresentam os intervalos de confiança de todas as variações. Ao analisar separadamente os blocos relativos aos cincos valores de $\mathrm{K}(5,10,15,20 \mathrm{e}$ 25) - separados pelas linhas vermelhas - , nota-se que as abordagens LSBR foram melhores que a UserKNN. Além disso, percebe-se que o desempenho da abordagem LSBR varia dependendo das dimensões de EA consideradas.

Desconsiderando o algoritmo UserKNN e blocando pelo valor de $\mathrm{K}$, foram executados testes estatísticos para confirmar o que previamente se acreditou com base na Figura 1: tanto os resultados da ANOVA (teste paramétrico) quanto do KruskalWallis H (teste não paramétrico) [Boslaugh e Watters 2008] indicaram que é possível rejeitar a hipótese de que não há diferença de resultado ao variar as dimensões consideradas na abordagens. Tendo em vista o objetivo deste estudo, isso implica dizer que as dimensões de EA influenciam de maneira diferente na recomendação personalizada de recursos educacionais para discentes. 
VI Congresso Brasileiro de Informática na Educação (CBIE 2017)

Anais do XXVIII Simpósio Brasileiro de Informática na Educação (SBIE 2017)

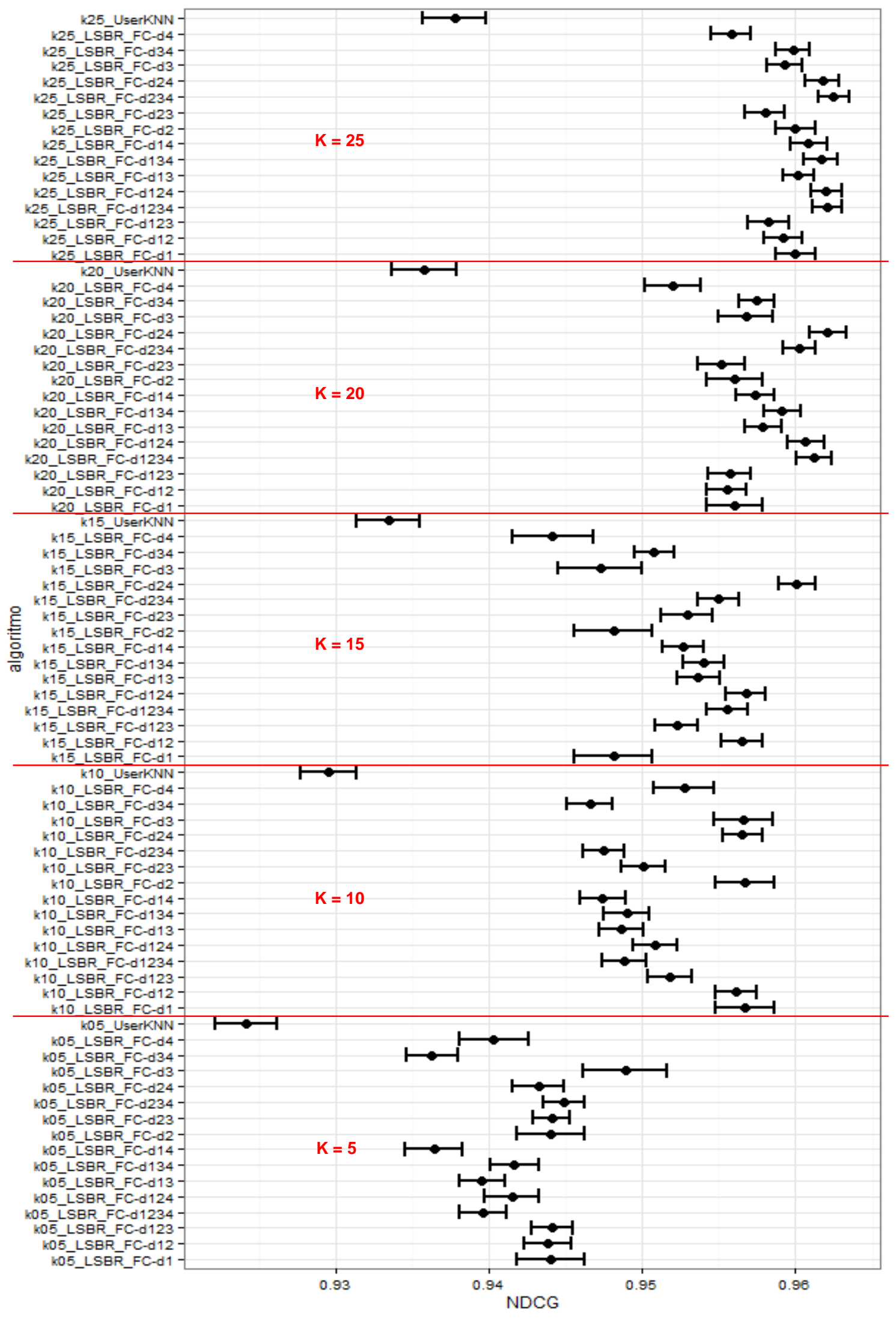

Figura 1. Intervalos de Confiança relativos aos algoritmos UserKNN e variações do LSBR_FC, considerando a métrica NDCG. 
VI Congresso Brasileiro de Informática na Educação (CBIE 2017)

Anais do XXVIII Simpósio Brasileiro de Informática na Educação (SBIE 2017)

Ressalta-se que os testes ANOVA e Kruskal-Wallis $\mathrm{H}$ foram realizados considerando as métricas AUC, MAP, P@5, R@5 e NDCG, sendo também gerados gráficos análogos à Figura 1 para as métricas AUC, MAP, P@5 e R@5. Todavia, como tais gráficos se mostraram, em geral, similares, além do aspecto de limitação de tamanho do artigo, foi decidido apresentar aqui uma análise considerando apenas a métrica NDCG (devido a ser a única, dentre as outras, a considerar a relevância dos itens recomendados e a sua posição na lista ordenada).

Ainda em relação ao objetivo deste estudo, a partir dos intervalos de confiança (Figura $1 \mathrm{e}$, mais detalhadamente, observando os intervalos de confiança em figuras como a Figura 2), não foi possível identificar a(s) dimensão(ões) com maior influência.

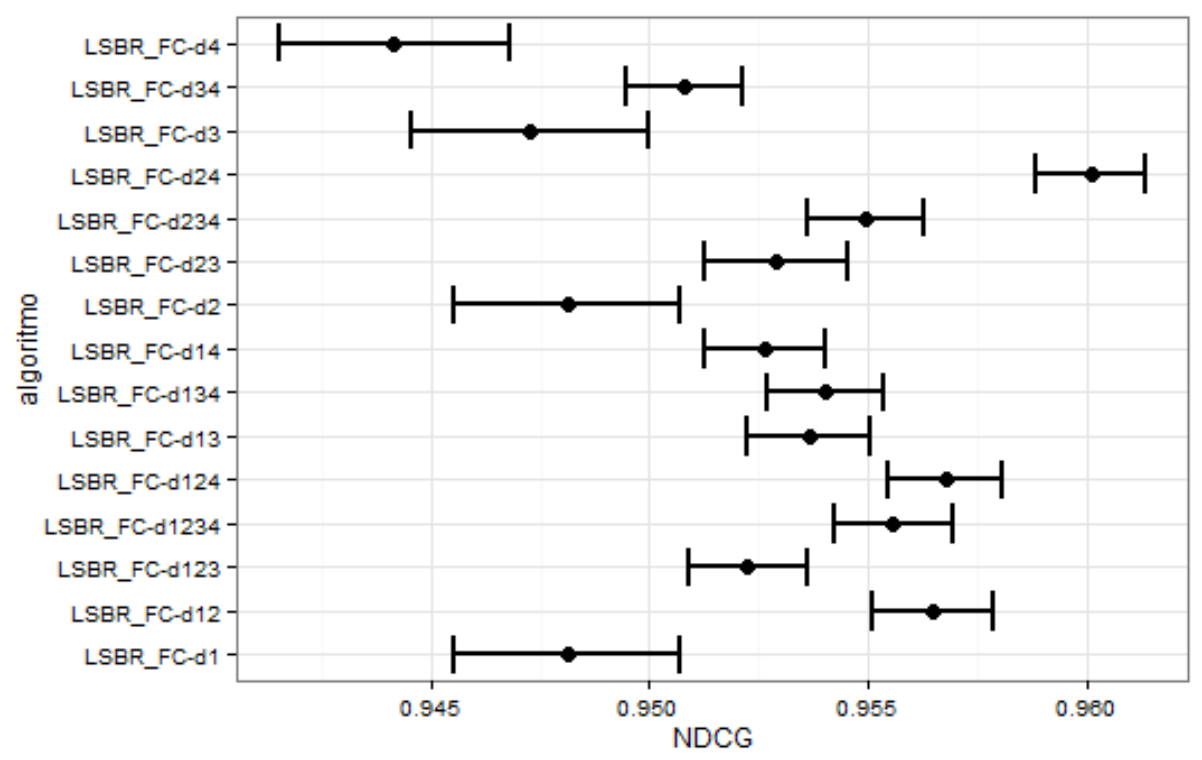

Figura 2. Intervalos de Confiança para as variações do LSBR_FC, considerando a métrica NDCG e K = 15.

Com o objetivo de descobrir a(s) dimensão(ões) de EA com maior influência no processo de recomendação, foram executados testes T (paramétrico) e Mann-Whitney U (não paramétrico) [Boslaugh e Watters 2008], par a par (pairwise), sendo planejada a geração de rankings (para cada bloco de valores de $\mathrm{K}$ ) com base nos resultados desses testes.

Para criar esses rankings, foi definida esta abordagem: (i) ordenação inicial das variações de LSBR com base na média da métrica NDCG; (ii) verificação dos resultados de ambos os testes ( $\mathrm{T}$ e Mann-Whitney $\mathrm{U}$ ) para o par de variações das posições adjacentes dessa ordenação inicial; e (iii) definição de posições diferentes do ranking (nos casos em que ambos os testes estatísticos indicaram diferença entre o par de variação) ou posições iguais (nos casos contrários).

Destaca-se que, apesar de haver alguns pares de variação estatisticamente diferentes (ao serem analisados todos os pares dos testes $T$ e Mann-Whitney U), não houve casos em que uma variação (ou um conjunto de variações) pudesse ser colocada em uma posição diferente das demais no ranking. Tal fato era esperado, uma vez que foram vistas várias sobreposições dos intervalos de confiança (especialmente visíveis em figuras como a Figura 2). Portanto, no Quadro 1, todas as variações ocupam a mesma posição no ranking, variando a ordem com base apenas na média. 
VI Congresso Brasileiro de Informática na Educação (CBIE 2017)

Anais do XXVIII Simpósio Brasileiro de Informática na Educação (SBIE 2017)

Quadro 1. Rankings de desempenho das variações de LSBR, considerando a métrica NDCG.

\begin{tabular}{|c|c|c|c|c|}
\hline$K=5$ & $K=10$ & $K=15$ & $K=20$ & $K=25$ \\
\hline$L S B R \_F C-d 3$ & $\left.L S B R \_F C-d 1\right)$ & LSBR_FC-d24] & LSBR_FC-d24 & LSBR_FC-d234 \\
\hline$L S B R \_F C-d 234$ & $L S B R_{-} F C-d 2$ & LSBR_FC-d124 & LSBR_FC-d1234 & LSBR_FC-d1234 \\
\hline$L S B R \_F C-d 23$ & $L S B R_{-} F C-d 3$ & LSBR_FC-d12 & LSBR_FC-d124 & LSBR_FC-d124 \\
\hline$L S B R_{-}^{-} F C-d 123$ & $L S B R_{-}^{-} F C-d 24$ & LSBR_FC-d1234 & LSBR_FC-d234 & LSBR_FC-d24 \\
\hline LSBR_FC-d1 & $L S B R_{-} F C-d 12$ & LSBR_FC-d234 & LSBR_FC-d134 & LSBR_FC-d134 \\
\hline LSBR_FC-d2 & $L S B R+F C-d 4 J$ & LSBR_FC-d134 & LSBR_FC-d13 & LSBR_FC-d14 \\
\hline LSBR_FC-d12 & LSBR_FC-d123 & LSBR_FC-d13 & LSBR_FC-d34 & LSBR_FC-d13 \\
\hline LSBR_FC-d24 & LSBR_FC-d124 & LSBR_FC-d23 & LSBR_FC-d14 & LSBR_FC-d1 \\
\hline LSBR_FC-d134 & LSBR_FC-d23 & LSBR_FC-d14 & LSBR_FC-d3 & LSBR_FC-d2 \\
\hline LSBR_FC-d124 & LSBR_FC-d134 & LSBR_FC-d123 & LSBR_FC-d1 & LSBR_FC-d34 \\
\hline LSBR_FC-d4 & LSBR_FC-d1234 & LSBR_FC-d34 & LSBR_FC-d2 & LSBR FC-d3 \\
\hline LSBR_FC-d1234 & LSBR_FC-d13 & LSBR FC-d1 & LSBR FC-d123 & LSBR FC-d 12 \\
\hline LSBR_FC-d13 & LSBR_FC-d234 & LSBR_FC-d2 & LSBR_FC-d12 & LSBR_FC-d123 \\
\hline LSBR_FC-d14 & LSBR_FC-d14 & LSBR_FC-d3 & LSBR_FC-d 23 & LSBR_FC-d23 \\
\hline LSBR FC-d34 & LSBR FC-d34 & LSBR FC-d4 & LSBR FC-d4 & LSBR FC-d4 \\
\hline
\end{tabular}

No Quadro 1, está realçada a variação d1234 (que considera todas as quatro dimensões do modelo Felder-Silverman), sendo destacadas em negrito as variações com resultados similares (isto é: nos testes par a par, o p-valor obtido para os que estão em negrito e o d1234 foi maior que o nível de significância de 0,05 , não sendo possível rejeitar a hipótese nula de que, em média, as variações são iguais em relação à métrica NDCG; portanto, não se considerou diferença entre os pares). Em itálico (e agrupadas por chaves) estão as variações que implicaram em maior acurácia na recomendação em comparação com a variação que considera todas as quatro dimensões do modelo. Isto é: pelos testes par a par, considerou-se diferença entre a variação d1234 e as variações em itálico.

A partir dos testes par a par, foi observado o seguinte: para $\mathrm{K}=5$, as variações $\mathrm{d} 3, \mathrm{~d} 234, \mathrm{~d} 23$ e d123 apresentaram-se melhores que d1234, que teve o $12^{\circ}$ maior valor médio; para $\mathrm{K}=10$, a variação $\mathrm{d} 1234$ teve o $11^{\circ}$ maior valor médio, apresentando desempenho inferior às variações $\mathrm{d} 1, \mathrm{~d} 2, \mathrm{~d} 3, \mathrm{~d} 24, \mathrm{~d} 12$ e $\mathrm{d} 4$; para $\mathrm{K}=15$, a variação d1234 teve o $4^{\circ}$ maior valor médio, sendo percebido que a variação d24 apresentou-se melhor que ela; para $\mathrm{K}=20$, a variação d1234 teve o $2^{\circ}$ maior valor médio, apresentando resultado similar às variações d24, d124, d234 e d134; para $\mathrm{K}=25$, a variação d1234 teve, novamente, o $2^{\circ}$ maior valor médio, apresentando desempenho similar às variações d234, d124, d24 e d134.

A partir da análise dos resultados apresentados, percebe-se a dificuldade em definir precisamente a(s) dimensão(ões) com maior influência na acurácia das recomendações baseadas em EA. Apesar dos rankings para $\mathrm{K}=5$ e $\mathrm{K}=10$ apresentarem algumas dimensões individuais com desempenho melhor que a combinação de todas as dimensões, isso não se repete com $\mathrm{K}$ igual a 15, 20 ou 25 (sendo, no entanto, a variação d1234 melhor que d1, d2, d3 e d4, nesses três casos). E ressalta-se aqui que, no geral, como percebe-se a partir da Figura 1, o desempenho das recomendações é melhor com $\mathrm{K}=25$ em comparação a $\mathrm{K}=5$, por exemplo. Portanto, defende-se que as dimensões individualmente $(\mathrm{d} 1, \mathrm{~d} 2, \mathrm{~d} 3$ e $\mathrm{d} 4)$ não tendem a ter desempenho melhor que a combinação de todas as dimensões (d1234).

É importante destacar que, nos casos analisados pelos testes par a par, estas combinações tiveram, pelo menos, o mesmo desempenho de d1234: d124, d134, d234 e 
d24. Destas, é possível destacar ainda mais a combinação d24 - a única considerada em dois casos $(K=10$ e $K=15)$ com desempenho melhor que d1234, além de contemplar suas dimensões em duas das outras três variações destacadas. Nesse sentido, sugere-se aprofundar o estudo, em outras bases de dados, a fim de reafirmar os resultados obtidos e confirmar a possibilidade de simplificar o algoritmo de recomendação baseado em EA, focando nas dimensões 2 (Percepção - Estilo Sensorial e Estilo Intuitivo) e 4 (Compreensão - Estilo Sequencial e Estilo Global).

\section{Considerações Finais}

Em relação aos resultados apresentados na Seção 4, notou-se inicialmente que, com a base de dados utilizada, as variações do algoritmo de recomendação baseada em EA foram melhores que o UserKNN. Em linhas gerais, visando responder às questões de pesquisa citadas na Seção 1, percebeu-se que as dimensões do modelo FelderSilverman influenciam diferentemente na recomendação personalizada de recursos educacionais para discentes, não sendo possível indicar precisamente quais dimensões poderiam ser desconsideradas para simplificar a utilização do modelo. Todavia, há indícios que a combinação das informações das dimensões Percepção e Compreensão pode influenciar para a obtenção de maior acurácia das recomendações em SRE.

Ressalta-se que o estudo descrito neste artigo possui limitação em relação à generalização dos resultados, uma vez que a base de dados utilizada considera apenas 55 alunos de uma mesma área (Computação). Todavia, este serviu para indiciar possibilidades de melhorar a acurácia de SRE a partir do conceito de EA.

A consideração da não totalidade do modelo Felder-Silverman ao construir o perfil do usuário em SRE é relevante, pois implicaria em menor tempo despendido pelos usuários para responder ao questionário ILS (menos questões necessárias) e, considerando sistemas automáticos de detecção, a simplificação implicaria em planejar/executar menos regras.

Embora a teoria de EA tenha adquirido grande influência no campo da Educação, sendo vasta a literatura sobre isso, não há um consenso sobre o valor de sua aplicação prática. Há pesquisadores céticos em relação à utilização da teoria de EA, a exemplo de Kirschner (2017) e Newton e Miah (2017), que indicam a falta de uma base de evidências para seu uso. De acordo com Pashler et al. (2009), poucos estudos utilizaram uma metodologia experimental capaz de testar a validade dos EA aplicados à Educação, não havendo evidências para justificar aplicar EA em sala de aula.

Em um estudo recente realizado com acadêmicos do Reino Unido [Newton e Miah 2017], foi apresentado que 58\% dos participantes acreditam que os EA são eficazes, embora apenas 33\% usam realmente tal conceito. Vale ressaltar que quase todos os participantes $(94,4 \%)$ que, no final da pesquisa, disseram que ainda usariam EA, concordaram inicialmente com esta afirmativa: "I try to organize my teaching to accommodate different student Learning Styles (e.g., visual, kinaesthetic, assimilator/converger)". Além disso, dos pesquisadores que indicaram que continuarão usando EA, 89\% deles concordaram com esta afirmativa: "Even though there is no 'evidence base' to support the use of Learning Styles, it is my experience that their use in my teaching benefits student learning". A partir disso, é possível comentar que, embora faltem nítidas evidências que comprovem a eficácia dos EA, muitos daqueles 
VI Congresso Brasileiro de Informática na Educação (CBIE 2017)

Anais do XXVIII Simpósio Brasileiro de Informática na Educação (SBIE 2017)

que utilizam tal teoria para organizar seu ensino acabam percebendo benefícios na aprendizagem dos alunos.

Ainda no âmbito das críticas em relação a EA, nota-se que um dos argumentos é de que não se percebe benefício em diagnosticar os EA dos alunos usando uma das classificações específicas e tentando combinar o ensino com esses estilos [Newton e Miah 2017]. Mesmo que não haja evidências de benefício relativo a combinar os EA com a metodologia de ensino empregada em sala de aula, os autores deste artigo defendem que, no contexto de SRE, é possível utilizar o conceito de EA visando realizar recomendações úteis - assim como apontam as pesquisas de Zaina et al. (2012), Resende e Dorça (2015) e Aguiar, Fechine e Costa (2015) —, sendo conveniente destacar que o conceito de EA pode ser empregado para resolver o problema de coldstart em SR.

Reforça-se, neste ponto, que o uso da teoria de EA, neste estudo, não é para diagnosticar como é cada aluno (por exemplo: "Fulano é Ativo, Sensorial, Visual e Sequencial" e "Sicrano é Reflexivo, Sensorial, Verbal e Global") e, baseando-se nisso, propor métodos de ensino específicos (o que é um aspecto objurgado por críticos em EA). Considerando que, de fato, os alunos têm particularidades, e visando à construção do perfil do usuário em SRE, notou-se que é útil considerar os EA como meio para identificar alunos com preferências similares (ou seja: respostas similares ao ILS) como forma de definir os vizinhos mais próximos, no processo de FC, diferentemente do processo mais tradicional (UserKNN).

Com base neste estudo, espera-se contribuir com a linha de pesquisa relativa a considerar a teoria de EA em SRE, objetivando uma utilização simplificada do modelo Felder-Silverman. Além disso, almeja-se, a partir deste artigo, que outros pesquisadores considerem a possibilidade de estudar e contribuir com a descoberta de evidências (ou não evidências) em se utilizar dimensões do modelo Felder-Silverman na construção de sistemas computacionais para promoção da aprendizagem.

\section{Referências}

Aggarwal, C. C. (2016). Recommender Systems: The Textbook. 1 ed. Springer International Publishing, 498 p., ISBN: 978-3-319-29659-3.

Aguiar, J. J. B.; Fechine, J. M.; Costa, E. B. (2014). Estilos Cognitivos e Estilos de Aprendizagem em Informática na Educação: um mapeamento sistemático focado no SBIE, WIE e RBIE. In: Anais do XXV Simpósio Brasileiro de Informática na Educação (SBIE/CBIE), Dourados, p. 441-450.

Aguiar, J. J. B.; Fechine, J. M.; Costa, E. B. (2015). Recomendação de Objetos de Aprendizagem baseada na Popularidade dos Objetos e nos Estilos de Aprendizagem dos Alunos. In: Anais do XXVI Simpósio Brasileiro de Informática na Educação (SBIE/CBIE), Maceió, p. 1147-1156.

Bativa, G. B.; Stiubiener, I. (2011). Ferramenta de Identificação de Perfis de Aprendizes FIPA. In: Anais do XXII Simpósio Brasileiro de Informática na Educação (SBIE), Aracaju, p. $880-883$.

Boslaugh, S.; Watters, P. A. (2008). Statistics in a Nutshell. O'Reilly Media. ISBN: 978-0-59651049-7.

Costa, E.; Aguiar, J.; Magalhães, J. (2013). Sistemas de Recomendação de Recursos Educacionais: conceitos, técnicas e aplicações. In: Anais da Jornada de Atualização em 
VI Congresso Brasileiro de Informática na Educação (CBIE 2017)

Anais do XXVIII Simpósio Brasileiro de Informática na Educação (SBIE 2017)

Informática na Educação (JAIE/CBIE), p. 57-78.

Elahi, M.; Braunhofer, M.; Ricci, F.; Tkalcic, M. (2013). Personality-Based Active Learning for Collaborative Filtering Recommender Systems. In: Baldoni, M.; Baroglio, C.; Boella, G.; Micalizio, R. (Eds.), Proceeding of the XIIIth International Conference on AI*IA 2013: Advances in Artificial Intelligence, v. 8249, p. 360-371, Springer.

Felder, R. M.; Silverman, L. K. (1988). Learning and Teaching Styles in Engineering Education. Journal of Engineering Education, v. 78, n. 7, p. 674-681.

Felder, R.; Soloman, B. A. (1999). Index of Learning Styles (ILS). Disponível em: $<$ http://www4.ncsu.edu/unity/lockers/users/f/felder/public/ILSpage.html>. Acesso em: 27 jun. 2017.

Gantner, Z.; Rendle, S.; Freudenthaler, C.; Schmidt-Thieme, L. (2011). MyMediaLite: A Free Recommender System Library. In: Proceedings of the 5th ACM Conference on Recommender Systems (RecSys 2011). Chicago, USA.

Han, J.; Kamber, M. (2006). Data Mining: Concepts and Techniques. Elsevier.

IEEE. (2002). IEEE Standard for Learning Object Metadata. IEEE Std 1484.12.1-2002, p. i-32.

Kirschner, P. A. (2017). Stop propagating the learning styles myth. Computers \& Education, v. 106(3), p. 166-171.

Klašnja-Milićević, A.; Vesin, B.; Ivanović, M.; Budimac, Z.; Jain, L. C. (2017). E-Learning Systems: Intelligent Techniques for Personalization. Springer International Publishing.

Newton, P. M.; Miah, M. (2017). Evidence-Based Higher Education - Is the Learning Styles "Myth" Important? Frontiers in Psychology, v. 8, 444.

Pashler, H.; McDaniel, M.; Rohrer, D.; Bjork, R. (2009). Learning Styles: Concepts and Evidence. Psychological Science in the Public Interest, v. 9, n. 3, p. 105-119.

Resende, D. T.; Dorça, F. A. (2015). Recomendação de conteúdo personalizada com base em estilos de aprendizagem: uma abordagem prática. Revista Brasileira de Informática na Educação (RBIE), v. 23, n. 3, p. 11-25.

Roshchina, A. (2012). TWIN: Personality-based Recommender System. MSc thesis. Tallaght Institute of Technology, Ireland.

Schunk, D. H. (2012). Learning theories: an educational perspective (6th ed.). Boston: Pearson.

Siemens, G. (2005). Connectivism: Learning as network-creation. ASTD Learning News, v. 10, n. 1, p. 1-28.

Truong, H. M. (2016). Integrating learning styles and adaptive e-learning system: Current developments, problems and opportunities. Computers in Human Behavior, v. 55, part B, p. 1185-1193.

Valaski, J.; Malucelli, A.; Reinehr, S. (2011). Revisão dos Modelos de Estilos de Aprendizagem Aplicados à Adaptação e Personalização dos Materiais de Aprendizagem. In: Anais do XXII Simpósio Brasileiro de Informática na Educação (SBIE), Aracaju, p. 844-847.

Xia, F.; Asabere, N. Y.; Liu, H.; Chen, Z.; Wang, W. (2014). Socially Aware Conference Participant Recommendation with Personality Traits. IEEE Systems Journal, p. 1-12.

Zaina, L. A.; Bressan, G.; Cardieri, M. A. A.; Rodrigues Junior, J. F. (2012). E-LORS: Uma abordagem para recomendação de Objetos de Aprendizagem. Revista Brasileira de Informática na Educação (RBIE), v. 20, n. 1, p. 4-16. 